\title{
Use of sofosbuvir in chronic kidney disease: Is it necessary?
}

\author{
Tae Seop Lim ${ }^{1,2,3}$, Sang Hoon Ahn ${ }^{1,2,3,4}$ \\ 'Department of Internal Medicine, ${ }^{2}$ Institute of Gastroenterology, Yonsei University College of Medicine, Seoul; ${ }^{3}$ Yonsei Liver Center, \\ Severance Hospital, Seoul; ${ }^{3}$ Brain Korea 21 Project for Medical Science, Seoul, Korea
}

Keywords: Sofosbuvir; Chronic hepatitis C; Chronic kidney disease; Renal function

\section{See Article on Page 316}

Hepatitis C virus (HCV) is one of the major causes of chronic liver disease around the world, and nearly 180 million people are thought to be infected with HCV chronically.' In Korea, the prevalence of chronic hepatitis $\mathrm{C}(\mathrm{CHC})$ is estimated at $0.78 \%{ }^{2}$ The main purpose of $\mathrm{HCV}$ eradication is to prevent progression of cirrhosis, liver-related complication, and HCC development. In addition to these main reasons to treat HCV infection, in patients with chronic kidney disease (CKD) who are potential candidates of kidney transplantation, there is an additional reason is to prevent HCV specific complications associated with kidney transplantation. Patients with HCV who undergo hemodialysis have a worse prognosis than dialysis patients without $\mathrm{HCV}^{3,4} \mathrm{~A}$ recent metaanalysis including 11,589 patients on hemodialysis resulted that HCV infection was an independent predictor of mortality in hemodialysis patients. ${ }^{5}$ Therefore, HCV eradication is mandatory in CKD patients with HCV for an additional indication, because they are candidates for kidney transplantation. ${ }^{6,7}$

Despite these hepatic and extrahepatic benefits of eradicat- ing HCV in CKD patients, traditionally accepted interferon based therapy in CKD patients has been unsatisfactory due to its suboptimal effects and toxicity, depending on the comorbid conditions and the extent of renal impairment. ${ }^{8,9}$ Therefore, eradicating HCV in CKD patients has been challenging. However, the emergence of direct-acting antivirals (DAAs) has changed the treatment trend of patients with CHC, and the decision to treat HCV in CKD patients has also been less challenging.

Sofosbuvir is one of the most widely used DAAs, and it is a highly effective nucleoside NS5B polymerase inhibitor. It should be administered at a dose of $400 \mathrm{mg}$ once a day, and it is combined with other DAAs, such as the NS5A inhibitor ledipasvir, the NS3 protease inhibitor simeprevir, or ribavirin. ${ }^{7}$ Sofosbuvir is mainly eliminated through kidney, so drug concentrations increase in patients with severe renal dysfunction. According to a clinical pharmacology study which enrolled patients without HCV, drug levels in those with $\mathrm{eGFR} \geq 30 \mathrm{~mL} / \mathrm{min}$ per $1.73 \mathrm{~m}^{2}$ were similar to those in people with normal renal function. ${ }^{10}$ On the contrary, the levels of drug were significantly increased in people with eGFR $<30 \mathrm{~mL} / \mathrm{min}$ per $1.73 \mathrm{~m}^{2}$ or on hemodialysis. ${ }^{10}$ Based on these results, in the United States, this drug was approved in Food and Drug Administration

\section{Abbreviations:}

CHC, chronic hepatitis $C_{;}$CKD, chronic kidney disease; DAA, direct-acting antivirals; $\mathrm{HCV}$, Hepatitis C virus

\section{Corresponding author : Sang Hoon Ahn}

Department of Internal Medicine, Yonsei University College of Medicine, 50-1 Yonsei-ro, Seodeamun-gu, Seoul 03722, Korea

Tel. +82-2-2228-1936, Fax. +82-393-6884

E-mail:AHNSH@yuhs.ac

http://orcid.org/0000-0002-3629-4624 
(FDA) in patients with eGFR $\geq 30 \mathrm{~mL} / \mathrm{min}$ per $1.73 \mathrm{~m}^{2},{ }^{10}$ and the current guideline recommends that adjustments of DAAs including sofosbuvir are not needed in patients with mild to moderate renal dysfunction (eGFR $\geq 30 \mathrm{~mL} / \mathrm{min}$ per $1.73 \mathrm{~m}^{2}$ ). ${ }^{1}$ However, in the real world, many clinicians have concerns to use sofosbuvir based regimens in CKD patients, because clinical data are limited and heterogenous. ${ }^{11,12}$

The study by Shin, et al. is the first real world study which evaluated efficacy and safety of diverse sofosbuvir-containing regimen in CKD stage 3 patients. ${ }^{13}$ They confirmed the current guideline that sofosbuvir-based regimen can be used in patients with mild to moderate renal impairment (eGFR $\geq 30 \mathrm{~mL} / \mathrm{min} / 1.73 \mathrm{~m}^{2}$ ). They focused efficacy and safety of sofobuvir-based therapy in $\mathrm{CHC}$ patients with renal impairment. The SVR rate at 12 weeks after end of the therapy was $85.7 \%$. Although greater than $30 \%$ decrease of eGFR was seen in 4 out of 28 patients, renal function was subsequently improved to normal in all 4 patients. This result suggests that sofosbuvir-based regimen could be used in CKD patients with close monitoring when it is needed. However, currently, there are other DAAs which can be used more safely for patients with renal dysfunction. An increasing evidence confirmed that elbasvir/grazoprevir regimen or ombitasvir/paritaprevir/ ritonavir with or without dasabuvir regimen can be used in CKD patients, even CKD stage 4 or 5 , with HCV genotype 1 or $4 .{ }^{14}$

Is sofosbuvir still necessary for treatment of $\mathrm{CHC}$ combined with CKD? Efficacy and safety of sofosbuvir-based therapy in CKD patients should be more evaluated because CKD patients with HCV genotype 2, 3, or an experience of DAA failures have limited treatment options except sofosbuvir-containing regimen. ${ }^{1.14}$

Although Shin, et al. showed efficacy and safety of sofobuvirbased regimen in patients with moderately impaired renal function, this study had potential limitations. There might be some bias to the study given its small sample size and retrospective nature. In addition, a comparative study should be required with elbasvir/grazoprevir regimen or ombitasvir/paritaprevir/ritonavir/ dasabuvir regimen in HCV genotype 1.

In conclusion, sofobuvir-based regimens can be used in patient with moderately impaired renal function without dose adjustments with close monitoring. A large prospective comparative study is warranted including various HCV genotypes and DAA failures.

\section{Authors' contribution}

Manuscript writing: Tae Seop Lim, Sang Hoon AhnFinal approval of manuscript: Sang Hoon Ahn

\section{Conflicts of Interest}

The authors have no conflicts to disclose.

\section{REFERENCES}

1. EASL recommendations on treatment of hepatitis C 2016. J hepatol 2017;66:153-194.

2. Kim DY, Kim IH, Jeong SH, Cho YK, Lee JH, Jin YJ, et al. A nationwide seroepidemiology of hepatitis $\mathrm{C}$ virus infection in South Korea. Liver int 2013;33:586-594.

3. Kalantar-Zadeh K, Kilpatrick RD, McAllister CJ, Miller LG, Daar ES, Gjertson DW, et al. Hepatitis C virus and death risk in hemodialysis patients. J Am Soc Nephrol 2007;18:1584-1593.

4. Nakayama E, Akiba T, Marumo F, Sato C. Prognosis of anti-hepatitis $C$ virus antibody-positive patients on regular hemodialysis therapy. J Am Soc Nephrol 2000;11:1896-1902.

5. Fabrizi F, Takkouche B, Lunghi G, Dixit V, Messa P, Martin P. The impact of hepatitis $C$ virus infection on survival in dialysis patients: meta-analysis of observational studies. J Viral Hepat 2007;14:697703.

6. Mathurin P, Mouquet C, Poynard T, Sylla C, Benalia H, Fretz C, et al. Impact of hepatitis $B$ and $C$ virus on kidney transplantation outcome. Hepatology 1999;29:257-263.

7. Suda G, Ogawa K, Kimura M, Nakai M, Sho T, Morikawa K, et al. Novel treatment of hepatitis $C$ virus infection for patients with renal impairment. J Clin Transl Hepatol 2016;4:320-327.

8. Fabrizi F, Dixit V, Messa P, Martin P. Interferon monotherapy of chronic hepatitis $C$ in dialysis patients: meta-analysis of clinical trials. J Viral Hepat 2008;15:79-88.

9. Gordon CE, Uhlig K, Lau J, Schmid CH, Levey AS, Wong JB. Interferon treatment in hemodialysis patients with chronic hepatitis $C$ virus infection: a systematic review of the literature and meta-analysis of treatment efficacy and harms. Am J Kidney Dis 2008;51:263-277.

10. Glead Siences Inc. These highlights do not include all the information needed to use SOVALDI safely and effectively. See full prescribing information for SOVALDI. <http://www.gilead.com/ /media/ Files/pdfs/medicines/liver-disease/sovaldi/sovaldi_pi.pdf>. Accessed 2017.08.02.

11. Saxena V, Koraishy FM, Sise ME, Lim JK, Schmidt M, Chung RT, et al. Safety and efficacy of sofosbuvir-containing regimens in hepatitis C-infected patients with impaired renal function. Liver int 2016;36:807-816

12. Bhamidimarri KR, Czul F, Peyton A, Levy C, Hernandez M, Jeffers L, et al. Safety, efficacy and tolerability of half-dose sofosbuvir plus simeprevir in treatment of Hepatitis $\mathrm{C}$ in patients with end stage renal disease. J Hepatol 2015;63:763-765.

13. Shin HP, Park JA, Burman B, Kozarek RA, Siddique A. Efficacy and safety of sofosbuvir-based regimens for treatment in chronic hepa- 
titis C genotype 1 patients with moderately impaired renal function. Clin Mol Hepatol 2017;23:316-322.

14. Mendizabal M, Reddy KR. Chronic hepatitis C and chronic kidney disease: Advances, limitations and unchartered territories. J Viral Hepat 2017;24:442-453. 